\title{
Cartesian products of directed graphs with loops
}

\author{
Wilfried Imrich* \\ Montanuniversität Leoben \\ Franz Josef-Straße 18, 8700 Leoben, Austria \\ imrich@unileoben.ac.at
}

\author{
Iztok Peterin ${ }^{\dagger}$ \\ University of Maribor, FEECS \\ Smetanova 17, 2000 Maribor, Slovenia \\ iztok.peterin@um.si
}

\begin{abstract}
We show that every nontrivial finite or infinite connected directed graph with loops and at least one vertex without a loop is uniquely representable as a Cartesian or weak Cartesian product of prime graphs. For finite graphs the factorization can be computed in linear time and space.
\end{abstract}

Keywords: Directed graph with loops; infinite graphs; Cartesian and weak Cartesian products Mathematics Subject Classifications: 05C25, 05C20,

\section{Introduction}

This note treats finite and infinite directed graphs with or without loops. It is shown that every connected, finite or infinite directed graph with at least one vertex without loop is uniquely representable as a Cartesian or weak Cartesian product of prime graphs, and that the factorization can be computed in linear time and space for finite graphs.

The note extends and unifies results by Boiko et al. 1] about the Cartesian product of finite undirected graphs with loops, and by Crespelle and Thierry [2] about finite directed graphs. For infinite graphs it generalises a result by Miller [8] and Imrich [6] about the weak Cartesian product.

Let us briefly mention that unique prime factorization with respect to the Cartesian product of connected finite graphs was first shown 1960 by Sabiduss 1 , and that Sabidussi also introduced the weak Cartesian product.

Sabidussi's proof is non-algorithmic. For undirected graphs the first factorization algorithm is due to Feigenbaum, Hershberger and Schäffer 4 . Its complexity is $O\left(n^{4.5}\right)$, where $n$ is the number of vertices of the graph. Subsequently the complexity was further reduced by a number of authors. The latest improvement, Imrich and Peterin [7, is linear in time and space in the number of edges.

For directed graphs the first factorization algorithm is from Feigenbaum [3] and assumes the undirected decomposition provided by [4. Crespelle and Thierry [2] also assume an undirected

* Partially supported by OEAD Projekt SI 08/2016.

${ }^{\dagger}$ Partially supported by the ARRS under the research grant P1-0297.

${ }^{1} 1963$ an independent proof was published by Vizing [10. 
decomposition and then compute the prime factorization of the directed graph in linear time and space. Here we present a considerably simpler algorithm of the same complexity and extend it to the case when loops are allowed. We use the same data structure as in [7] and wish to remark that a slight variation of the algorithm in 7 . would also allow a direct computation of the prime factors of connected directed graphs (with or without loops) in linear time.

\section{Preliminaries}

A directed graph $G$ with loops consists of a set $V(G)$ of vertices together with a subset $A(G)$ of $V(G) \times V(G)$. The elements of $A(G)$ are called arcs and are ordered pairs of vertices. If $a b$ is an arc, we call $a$ its origin, $b$ its terminus, and also refer to $a$ and $b$ as endpoints.

We allow that $a$ equals $b$. In this case we speak of a loop at vertex $a$ and say the vertex $a$ is looped. Notice that it is possible that $A(G)$ contains both $a b$ and $b a$.

To make the notation better readable we often write $v \in G$ instead of $v \in V(G)$, and $e \in G$ instead of $e \in A(G)$ or $E(G)$.

We introduce the notation $\overrightarrow{\Gamma_{0}}$ for the class of directed graphs with loops. To every graph $G \in \overrightarrow{\Gamma_{0}}$ we also define its shadow $\mathcal{S}(G)$. It has the same vertex set as $G$ and its set of edges $E(G)$ consists of all unordered pairs $\{a, b\}$ of distinct vertices for which $a b, b a$ or both are in $A(G)$. To indicate that $\{a, b\}$ is an edge, we will use the notation $[a, b]$, or simply ab. We say $G$ is connected if $\mathcal{S}(G)$ is connected, and set the distance $d_{G}(u, v)$ between two vertices, and the degree $d_{G}(u)$ of a vertex, equal to $d_{\mathcal{S}(G)}(u, v)$, resp. $d_{\mathcal{S}(G)}(u)$. The minimum degree of $G$ is denoted by $\delta$. We also use the notation $\Gamma$ for the class of simple graphs, $\Gamma_{0}$ for the class of simple graphs with loops, and $\vec{\Gamma}$ for the class of directed graphs without loops.

The Cartesian product $G \square H$ of graphs in $\overrightarrow{\Gamma_{0}}$ is defined on the Cartesian product $V(G) \times V(H)$ of the vertex sets of the factors. Its set of arcs is

$$
A(G \square H)=\{(x, u)(y, v) \mid x y \in A(G) \text { and } u=v, \text { or, } x=y \text { and } u v \in A(H))\} .
$$

If $G$ and $H$ have no loops, then this is also the case for $G \square H$. To obtain the definition of the Cartesian product of undirected graphs, one just replaces $A(G)$ by $E(G)$. Hence, the new definition generalizes the definition of the Cartesian product of simple graphs, directed graphs, and simple graphs with loops. Note that $\mathcal{S}(G \square H)=\mathcal{S}(G) \square \mathcal{S}(H)$.

Clearly Cartesian multiplication is commutative and the trivial graph $K_{1}$ is a unit. It is well known that it is associative in $\Gamma$. That it is associative in $\vec{\Gamma}$ and $\Gamma_{0}$ was shown in [3], resp. [1], for finite graphs. We defer the proof that this also holds for finite graphs in $\vec{\Gamma}_{0}$ to Section 4 where we show associativity in $\vec{\Gamma}_{0}$ with respect to the Cartesian and the weak Cartesian product.

A nontrivial, connected graph $G$ with at least one unlooped vertex is called irreducible or prime with respect to Cartesian multiplication if, for every factorization $G=A \square B$, either $A$ or $B$ has only one vertex. Every finite connected graph in $\vec{\Gamma}_{0}$ with at least one unlooped vertex is uniquely representable as a Cartesian product, up to the order and isomorphisms of the factors. Again we defer the proof and show in Section 5 that unique factorization holds for finite graphs with respect to the Cartesian product and for infinite graphs with respect to the Cartesian or the weak Cartesian product. 
For our proofs and algorithms projections and layers play an important role. The $i^{\text {th }}$ projection $p_{i}: V(G) \rightarrow V\left(G_{i}\right)$ of a product $\prod_{i=1}^{k} G_{i}$ is defined by $\left(v_{1}, \ldots, v_{k}\right) \mapsto v_{i}$ and the $G_{i}$-layer $G_{i}^{v}$ through a vertex $v \in G$ is the subgraph induced by the set

$$
\left\{w \in V(G) \mid w_{j}=v_{j} \text { for all } j \neq i, 1 \leq j \leq k\right\} .
$$

Sometimes we will also use the notation $p_{G_{i}}$ instead of $p_{i}$.

For unlooped graphs the projections $p_{i}: G_{i}^{v} \mapsto G_{i}$ are isomorphism, but this does not hold for graphs with loops unless $G_{i}^{v}$ contains an unlooped vertex, see the left part of Figure 1. The other part of the figure shows that directed graphs with no unlooped vertex need not have unique prime factorizations.
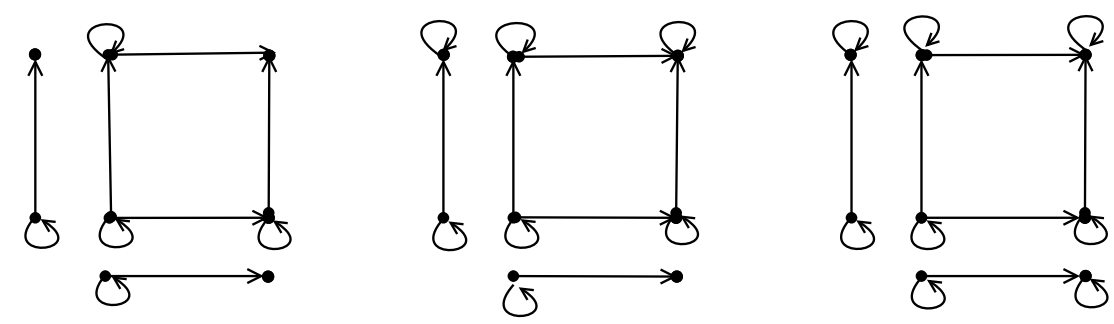

Figure 1: Cartesian products of graphs with loops

Often we color the edges or arcs of a graph and denote the color of $u v$ by $c(u v)$. For example, we usually color a product $\prod_{i=1}^{k} G_{i}$ by $k$ colors such that the edges of the $G_{i}$-layers are assigned color $i$.

We also need the fact that layers are convex, where a subgraph $H$ of a graph $G$ is called convex if any shortest path $P$ in $G$ between two vertices of $H$ is already in $H$. Another important property we formulate as a lemma.

Lemma 1 Let $G=G_{1} \square G_{2}$ be the product of two connected graphs in $\Gamma$, and $u, v \in V(G)$. Then there exists a unique vertex $x \in G_{1}^{u}$ of shortest distance from $v$ to $G_{1}^{u}$, and to any vertex $y \in G_{2}^{v}$ there is a shortest $v, y$-path that contains $x$.

Proof Let $u=\left(u_{1}, u_{2}\right)$ and $v=\left(v_{1}, v_{2}\right)$. Then $x=\left(v_{1}, u_{2}\right)$ is the unique vertex of $G_{2}^{v} \cap G_{1}^{u}$. By the Distance Formula [5, Lemma 5.2]

$$
d_{G}(a, b)=d_{G_{1}}\left(a_{1}, b_{1}\right)+d_{G_{2}}\left(a_{2}, b_{2}\right)
$$

for any $a, b \in G_{1} \square G_{2}$. Because $x_{1}=v_{1}$ and $x_{2}=y_{2}$ we have $\left.d_{G}(v, x)+d_{G}(x, y)\right)=d_{G_{1}}\left(v_{1}, x_{1}\right)+$ $d_{G_{2}}\left(v_{2}, x_{2}\right)+d_{G_{1}}\left(x_{1}, y_{1}\right)+d_{G_{2}}\left(x_{2}, y_{2}\right)=d_{G}(v, y)$. Therefore $x$ is on a shortest $v, y$-path for any $y \in G_{2}^{v}$.

This also means that every vertex $y \in G_{2}^{v}$ that is different from $x$ must have larger distance from $v$ than $x$. Hence $x$ is the unique vertex of shortest distance from $v$ in $G_{1}^{u}$.

It will be convenient to call the vertex $x$ of shortest distance from a vertex $v$ to a layer $G_{i}^{u}$ the projection of $v$ into $G_{i}^{u}$ and to denote it by $p_{G_{i}^{u}}(v)$. By the projection of an arc $u v$ we mean 
$p_{G_{i}^{u}}(u v)=p_{G_{i}^{u}}(u) p_{G_{i}^{u}}(v)$, which may be an arc or not, and if it is an arc, the orientation is not necessarily the same as that of $u v$. If $u w \in \mathcal{S}(G)$ and if the restriction of the projection $p_{G_{i}^{u}}$ to $u w$ and $p_{G_{i}^{u}}(u w)$ is not an isomorphism in $G$, then we call the pair $u w, p_{G_{i}^{u}}(u w)$ inconsistently directed.

If $v u$ and $v w$ are in different layers with respect to a factorization $G_{1} \square G_{2}$, say $v u \in G_{1}^{v}$ and $v w \in G_{2}^{v}$, then the vertex $x=p_{G_{1}^{w}}(u)=p_{G_{2}^{u}}(w)$, together with $v u w$ induces a square $v u x w$ without diagonals. It is the only square containing $v, u$, and $w$ and called product square. We call a product square vuxw in $\mathcal{S}\left(G_{1} \square G_{2}\right)$ consistently oriented if the arc or arcs between $v$ and $u$ and between $w$ and $x$ have the same orientation as the ones between $p_{G_{1}^{u}}(v)=p_{G_{1}^{u}}(w)$ and $p_{G_{1}^{u}}(u)=p_{G_{1}^{u}}(x)$, and similarly, the arc or arcs between $v$ and $w$ and between $u$ and $x$ have the same orientation as the ones between $p_{G_{2}^{w}}(v)=p_{G_{2}^{w}}(u)$ and $p_{G_{2}^{w}}(w)=p_{G_{2}^{w}}(x)$. It means that opposite edges, say $v u, w x$, represent either arcs $v u, w x$, or arcs $u v$ and $x w$, or arcs in both directions.

\section{Algorithms}

In this section we present two algorithms. The first one computes the prime factorization of a connected graph $G \in \vec{\Gamma}$ from a given prime factorization of $\mathcal{S}(G)$, the second computes the prime factorization of a connected graph $G \in \overrightarrow{\Gamma_{0}}$, where $G$ has at least one unlooped vertex, from a given prime factorization of $\mathcal{N}(G)$, where $\mathcal{N}(G)$ denotes the graph obtained from $G$ by the removal of the loops. Both algorithms are linear in the number of arcs.

The data structures that we use are incidence and adjacency lists. The incidence list of a graph in $\Gamma$ lists to every edge $e=[u, v]$ its endpoints, whereas the adjacency list consists of the lists of neighbors $N(v), v \in V(G)$. Every edge $e=[u, v]$ appears in $N(v)$ and $N(u)$. To both of these lists we add a pointer to the place of $e$ in the incidence list, and in the incidence list we add pointers to the place of $e$ in the lists $N(v)$ and $N(u)$.

For graphs $G$ in $\vec{\Gamma}$ we form the lists for $\mathcal{S}(G)$ and then indicate in the incidence list for every $[u, v]$ whether it is the shadow of $u v, v u$, or both $u v$ and $v u$. For loops we make an entry in the adjacency list, because $v$ is a neighbor of itself if it is looped. Clearly the space requirement is $O(|E(G)|)$, resp. $O(|A(G)|)$ in the directed case.

We also use a BFS-ordering of the vertices of $G$ with respect to a root $v_{0}$. It consists of the sets $L_{i}$ that contain the vertices of distance $i$ from $v_{0}$. Furthermore, the vertices of $G$ are enumerated by BFS-numbers that satisfy the condition that $\operatorname{BFS}(v)>\operatorname{BFS}(u)$ if the distance from $v_{0}$ to $v$ is larger than the one from $v_{0}$ to $u$. Layers through $v_{0}$ are called unit-layers and vertices of unit-layers are called unit-layer vertices.

If $v u$ is an edge or an arc, then we call $u$ a down-, cross-, or up-neighbor of $v$ if, respectively, $u$ is in a lower $L_{i}$-level than $v$, in the same, or in a higher $L_{i}$-level. For a given BFS-ordering we also subdivide every list of neighbors of a vertex $v$ into lists of down-, cross- or up-neighbors. For graphs in $\Gamma$ we refer to these lists as list of down-, cross- or up-edges, and remark that we will not need the list of up-neighbors, resp. up-edges, in our algorithms.

If $\mathcal{S}(G)=\prod_{j \in J} Z_{j}$ we color the edges of $\mathcal{S}(G)$ as described before with $i$ colors and subdivide every list of down- or cross-neighbors of a vertex $v$ into sublists of different colors, according to the color of $v u$. Furthermore, because for any subset of down- or cross-edges of a vertex $v$ with color $j$

${ }^{2}$ Clearly $\{x\}=G_{1}^{w} \cap G_{2}^{u}$. 
the projection $p_{Z_{j}^{v_{0}}}(v)$ is a natural bijection into the set of down-, resp. cross-edges of $p_{Z_{j}^{v_{0}}}(v)$ in $Z_{j}^{v_{0}}$, we use the same order in both lists.

To describe an edge $e$ we thus need a vertex $v$ of which it is a down-, or cross edge, the fact whether it is a down-or cross-edge, its color $c(e)$, and its number in the sublist of color $c(e)$. Given the place of $v$ in the adjacency list, the color of $e$ and its sublist number, we can then find the place of $e$ in the adjacency list in constant time. Furthermore, if we know the coordinates of $v$, then we can find the place of $v$ in the adjacency list in time proportional to the number of coordinates in which $v$ differs from $v_{0}$.

By Lemma 1 the projection $p_{Z_{i}^{v_{0}}}(v)$ is always closer to $v_{0}$ than $v$, unless $v$ already is a vertex of $Z_{i}^{v_{0}}$.

Let $\prod_{i=1}^{k} G_{i}$ be the prime factorization of a nontrivial, connected graph $G \in \vec{\Gamma}$. Then $\mathcal{S}(G)=$ $\prod_{i=1}^{k} \mathcal{S}\left(G_{i}\right)$, where the factors $\mathcal{S}\left(G_{i}\right)$ need not be prime. Let $\mathcal{S}\left(G_{i}\right)=H_{i, 1} \square \cdots \square H_{i, \ell(i)}$ be their prime factorizations. Then

$$
\mathcal{S}(G)=\prod_{i=1}^{k} \prod_{j=1}^{\ell(i)} H_{i, j}
$$

is a representation of $\mathcal{S}(G)$ as a Cartesian product of prime graphs. Because the prime factorization is unique, it is the prime factorization of $\mathcal{S}(G)$, up to the order and isomorphisms of the factors. In other words, for any prime factorization $\prod_{j \in J} Z_{j}$ of $\mathcal{S}(G)$, there is a partition $J=J_{1} \cup \cdots \cup J_{k}$ such that $\mathcal{S}\left(G_{i}\right)=\prod_{j \in J_{i}} Z_{j}$.

To find this partition we begin with the partition where the $J_{i}$ are one-element sets. Then we combine selected sets $J_{i}$ until we arrive at the desired final partition. The other partitions are called temporary partitions.

To keep track of these operations, we create a pointer $t_{c}$ with $t_{c}: r \rightarrow i$ if $r \in J_{i}$. We begin with the trivial partition of $J$ into one-element sets. Whenever we move from temporary partition $J_{1} \cup \cdots \cup J_{k}$ of $J$ to a new one, say $J_{1}^{\prime} \cup \cdots \cup J_{k^{\prime}}^{\prime}$, by combining some of the $J_{i}$, we update the pointers. We also assign the temporary color $t_{c}(i)$ to the edges in the $G_{i}^{\prime}$-layer of $\mathcal{S}(G)$, where $\mathcal{S}\left(G_{i}^{\prime}\right)=\prod_{j \in J_{i}^{\prime}} Z_{j}$. Note that $t_{c}$ produces the temporary color of any edge in constant time.

To update $t_{c}$ when we combine two colors, at most $|J|$ pointers have to be reset, each at constant cost. Because there are only $|J|$ colors, the total cost is $O\left(|J|^{2}\right)$. Recall that $|J|$ is the original number of factors. Because every vertex meets a layer of every one of the factors, we cannot have more factors than the minimum degree $\delta$ of $G$. So $O\left(|J|^{2}\right)=O\left(\delta^{2}\right)=O(n \delta)=O(m)$.

Algorithm 1 specifies when colors are combined. We have prove the correctness of the algorithm and to investigate its complexity.

Correctness of Algorithm 1 In $L_{1}$ all vertices are unit-layer vertices and all edges coincide with their projection into the unit-layers of their color. Hence all pairs $v u$ and $p_{X}(v) p_{X}(u)$, where $v \in L_{1}$ and $v u$ a down- or cross-edge, are consistently directed. Let this be the case for all pairs $v u$ and $p_{X}(v) p_{X}(u)$ where $v$ is in $L_{0} \cup \cdots \cup L_{k-1}$.

Suppose $v \in L_{k}$ and the algorithm detects a down- or cross-edge $v u$ of $v$ of temporary color $i$ for which $v u$ and its projection into $X=\left(\prod_{j \in J_{i}} Z_{j}\right)^{v_{0}}$ are not consistently directed. Let $Y=$ $\prod_{j \in J \backslash J_{i}} Z_{j}$ and $P$ be a shortest path from $v$ to $p_{X}(v)$. Clearly $P \in Y^{v}$, and the temporary color of all edges of $P$ is different from that of $v u$.

Let $P^{\prime}$ be the projection of $P$ into $Y^{u}$. Then the vertices of $P$, together with those of $P^{\prime}$, induce a subgraph $L$ of $\mathcal{S}(G)$ that is isomorphic to the product $P \square[u, v]$. We call it a ladder and the 


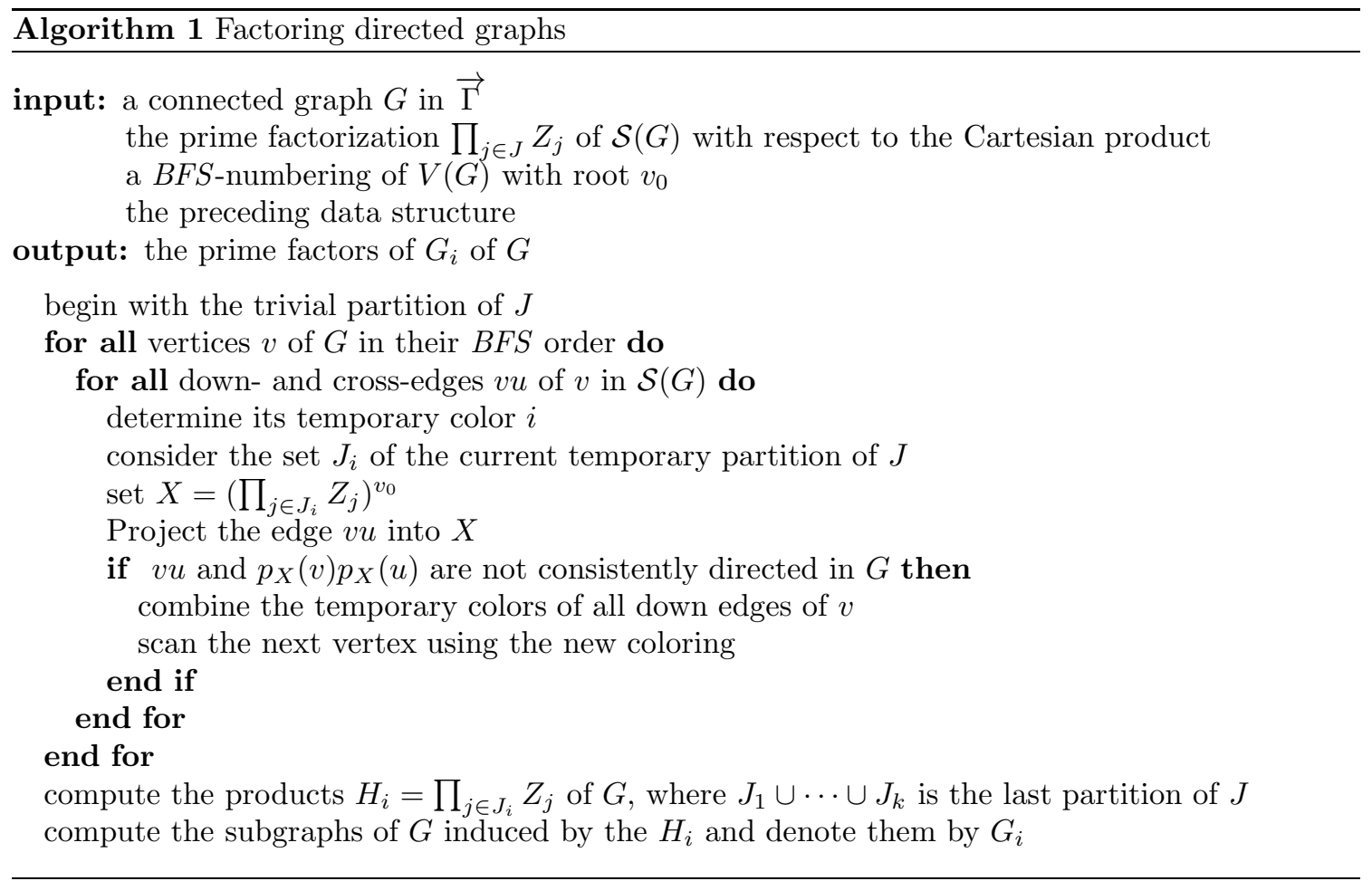

edges of $L$ that are not in $P \cup P^{\prime}$ rungs. All rungs $a b$ project into $p_{X}(v u)$, and, with the exception of the pair $\left\{v u, p_{X}(v u)\right\}$, all pairs $\left\{a b, p_{X}(v u)\right\}$ are consistently directed, because $a$ is in some $L_{j}$ with $j<k$. If $v^{\prime} u^{\prime}$ denotes the rung closest to $v u$, then $v v^{\prime} u^{\prime} u$ is a product square, because $v u$ and $v v^{\prime}$ have different colors. But then $v v^{\prime} u^{\prime} u$ is an inconsistently directed product square and we have to combine the colors of its edges.

Now, let $v q$ be any down-edge of $v$, whose temporary color $t_{c}(v q) \neq i$. Then there is a shortest path $Q$ from $v$ to $p_{X}(v)$ that contains $q$, and hence the color of $v q$ must be combined with that of $v u$.

When the algorithm terminates, all projections are consistently oriented. We thus arrive a factorization of $G$.

We still have to show that the $G_{i}$ are prime, that is, that we have not merged colors unnecessarily. Notice that we have only merged colors of inconsistently oriented product squares. Originally we had the product squares of the decomposition $\prod_{j \in J} Z_{j}$ of $\mathcal{S}(G)$. Since our operations only combined colors of edges in inconsistently directed squares, every single combination of colors was forced.

Complexity of Algorithm 1 The projection of a vertex $v$ into $X=\left(\prod_{j \in J_{i}} Z_{j}\right)^{v_{0}}$ has at most $\left|J_{i}\right|$ coordinates in $\prod_{j \in J} Z_{j}$ that are different form those of $v_{0}$, hence $p_{X}(v)$ can be computed in $O\left(\left|J_{i}\right|\right)=O(\delta)$ time. To find the edge $p_{X}(v u)$ we then need its type (down- or cross-edge), its color (original color) and its sublist number, which we inherit from $v u$. Then we can find $p_{X}(v u)$ in constant time and check in constant time whether the pair $v u, p_{X}(v u)$ is consistently directed. Since the number of down-edges is bounded by $d(v)$ the time complexity for each $v$ is thus $O(\delta)+O(d(v))$, and for all vertices together it requires $O\left(\sum_{v \in V(G)}(\delta+d(v))\right)=O(m)$ time. 
For the complexity of keeping track of the colorings, let us recall that we have to combine colors at most $|J|$ times and that every merging operation of two colors costs $O(|J|)$ time, hence the overall cost is $O\left(|J|^{2}\right)$, of which we already know that it is $O(m)$.

Theorem 2 Let $G$ be a connected, directed graph. Given a prime factorization of the shadow $\mathcal{S}(G)$ of $G$ with respect to the Cartesian product, one can compute the prime factorization of $G$ with respect to the directed product in $O(|A(G)|$ time.

Proof Given the adjacency lists of the prime factors of $\mathcal{S}(G)$ we can compute the data structure that we need for Algorithm 1 in time and space that is linear in the number of arcs. If the factors are given, say, via their adjacency matrices, we can still find their adjacency lists in linear time and then continue in linear time and space.

If the factorization of $\mathcal{S}(G)$ is computed by the algorithm of Imrich and Peterin [7, then one can use the data structure provided by that algorithm.

We continue with the prime factorization of connected, directed graphs with loops.

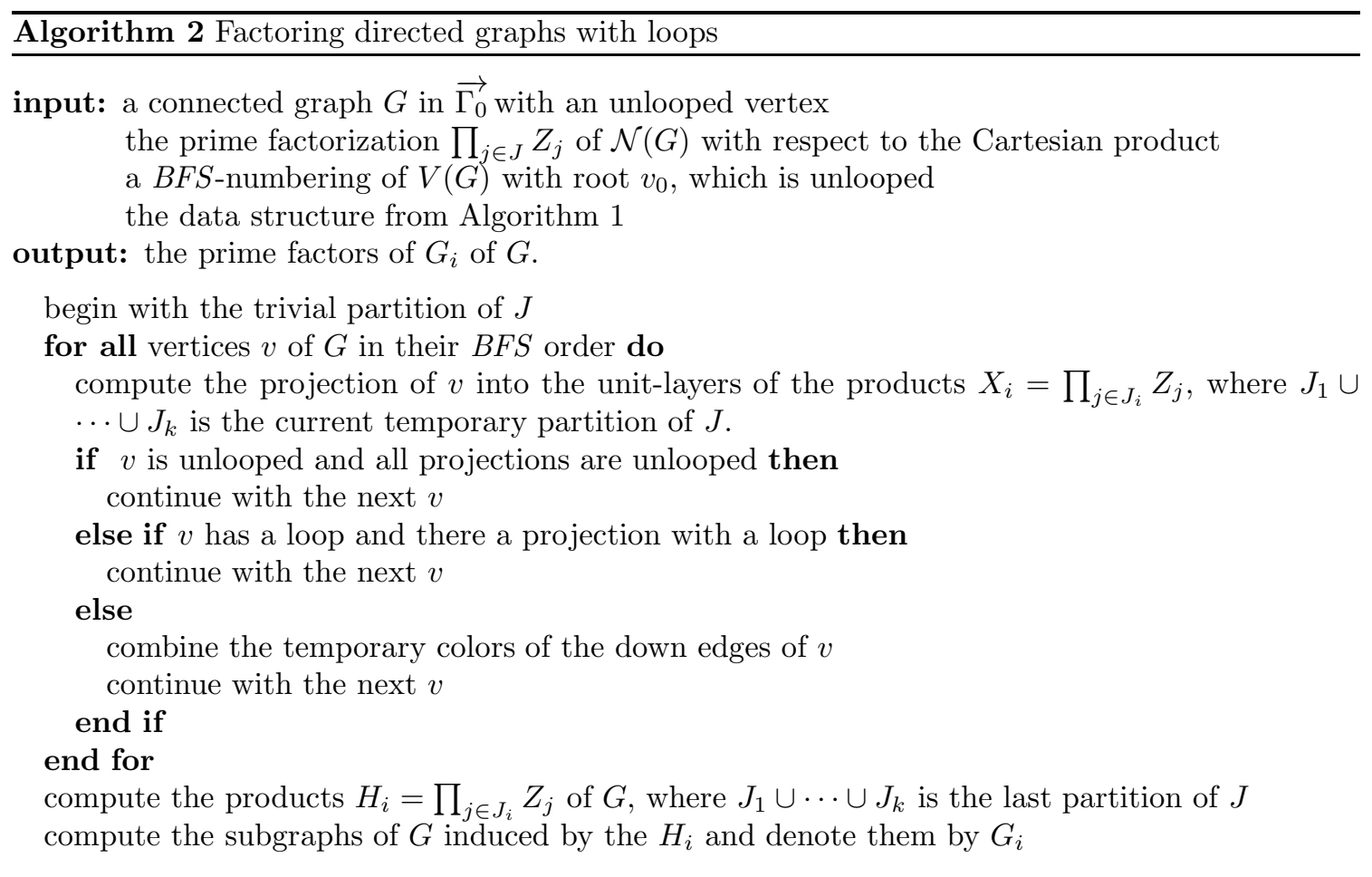

Correctness of Algorithm 2 If the projection of an unlooped vertex $v$ into a unit layer (with respect to an unlooped root) has a loop, then $v$ must be contained in that layer, and hence all shortest paths form $v$ to the projection of $v$. As in the correctness argument of Algorithm 1 this means that the colors of all down-edges of $v$ have to be combined.

Suppose all projections of a vertex with a loop are unlooped. Consider all projections that are different from $v_{0}$ and the respective unit-layers, that is, the layers $X_{i}^{v_{0}}=\left(\prod_{j \in J_{i}} Z_{j}\right)^{v_{0}}$, where the 
$J_{i}$ correspond to the temporary colors of the down-edges of $v$. Let $J^{\prime}$ be the set of the indices of these $J_{i}$ and form, for any proper subset $J^{\prime \prime}$ of $J^{\prime}$, the product $X=\prod_{j \in J^{\prime \prime}} X_{j}$. Then the projection of $v$ into $X^{v_{0}}$ is unlooped. This means, unless we combine all colors of the down-edges of $v$ we will only have unlooped projections.

Complexity of Algorithm 2 The computation of the projection of a vertex $v$ into $\left(\prod_{j \in J_{i}} Z_{j}\right)^{v_{0}}$ takes $O\left(\left|J_{i}\right|\right)$ time. The cost of computing them all is thus $O\left(\sum_{i=1}^{k}\left|J_{i}\right|\right)=O(|J|)=$ $O(\delta)$, which gives a total of $O(n \delta)=O(m)$ for all vertices together.

The complexity of keeping track of the colorings is $O(m)$, as in Algorithm 1 .

We have thus shown the following theorem.

Theorem 3 Let $G$ be a connected, directed graph with loops. Given a prime factorization of $\mathcal{N}(G)$ with respect to the Cartesian product, one can compute the prime factors of $G$ with respect to the Cartesian product in $O(|A(G)|$ time.

\section{The weak Cartesian product}

We consider infinite graphs now and begin with the definition of the Cartesian product of infinitely many factors.

Definition 1 Let $G_{\iota}, \iota \in I$, be a collection of graphs in $\overrightarrow{\Gamma_{0}}$. Then the Cartesian product

$$
G=\prod_{\iota \in I} G_{\iota}
$$

has as its set of vertices $V(G)$ all functions

$$
v: I \rightarrow \bigcup_{\iota \in I} V\left(G_{\iota}\right)
$$

with the property that $v(\iota) \in V\left(G_{\iota}\right)$. We call $v(\iota)$ the $\iota$-coordinate of $v$ and also denote it by $v_{\iota}$.

The set $A(G)$ of arcs of $G$ consists of all ordered pairs uv for which there exists a $\kappa$ such that $u_{\kappa} v_{\kappa} \in A\left(G_{\kappa}\right)$ and $u_{\iota}=v_{\iota}$ for all $\iota \in I \backslash\{\kappa\}$.

Furthermore, $v \in V(G)$ has a loop if at least one $v_{\iota}, \iota \in I$, has a loop in $G_{\iota}$.

This definition is equivalent to the definition of the Cartesian product for two factors as given in Section 2. To see this, observe that $V\left(G_{1} \square G_{2}\right)=\left\{\left(v_{1}, v_{2}\right) \mid v_{1} \in V\left(G_{1}\right), v_{2} \in V\left(G_{2}\right)\right\}$ by the old definition. Representing the ordered pairs $\left\{\left(v_{1}, v_{2}\right) \mid v_{1} \in V\left(G_{1}\right), v_{2} \in V\left(G_{2}\right)\right\}$ by the set of functions $v: I \rightarrow V\left(G_{1}\right) \cup V\left(G_{2}\right)$, where $v_{1} \in V\left(G_{1}\right)$ and $v_{2} \in V\left(G_{2}\right)$, it becomes clear that the definitions are equivalent.

Furthermore, let $n$ be a positive integer and $J_{1} \cup \cdots \cup J_{k}$ is an arbitrary partition of the set of integers between 1 and $n$, then there clearly is a natural isomorphism between $\prod_{i=1}^{n} V\left(G_{i}\right)$, the product

$$
\prod_{j=1}^{k} \prod_{i \in J_{k}} V\left(G_{i}\right)
$$


and the set of functions $v: I \rightarrow \bigcup_{i=1}^{n} V\left(G_{i}\right)$, where $v_{i} \in V\left(G_{i}\right)$. This means that Cartesian multiplication of finitely many sets is associative.

For infinite $I$ and any of its partitions $\left\{J_{\lambda} \mid \lambda \in \Lambda\right.$ \}e only have the isomorphism between $\prod_{\lambda \in \Lambda} \prod_{\iota \in J_{\lambda}} V\left(G_{\iota}\right)$ and the set of functions $v: I \rightarrow \bigcup_{\iota \in I} V\left(G_{\iota}\right)$ where $v(\iota) \in V\left(G_{\iota}\right)$, but the Cartesian product of infinitely many sets is still associative.

The fact that the set of factors need not be ordered in Definition 1 reflects the fact that Cartesian multiplication is commutative.

Lemma 4 Cartesian multiplication of directed graphs with loops is associative.

Proof Let $G_{\iota}, \iota \in I$, be a collection of directed graphs with loops, $G=\prod_{\iota \in I} G_{\iota},\left\{J_{\lambda} \mid \lambda \in \Lambda\right\}$ an arbitrary partition of $I$ and

$$
H=\prod_{\lambda \in \Lambda} \prod_{\iota \in J_{\lambda}} G_{\iota} .
$$

We have to show that $G \cong H$. To see this recall that there is a natural bijection between the vertices of $G$ and $H$. We will thus use the same notation for the vertices of $G$ and $H$.

Consider an arc $u v$ in $G$. All coordinates of $u$ and $v$ are identical, except for one, say $u_{\kappa} \neq v_{\kappa}$, and $u_{\kappa} v_{\kappa} \in A\left(G_{\kappa}\right)$. Let $\kappa \in J_{\lambda^{\prime}}$ and let $u_{J_{\lambda}}$ be the vertex in $\prod_{\iota \in J_{\lambda}} G_{\iota}$ with the coordinates $u_{\iota}$, where $\iota \in J_{\lambda}$. Analogously we define the $v_{J_{\lambda}}$. Then $u_{J_{\lambda}}=v_{J_{\lambda}}$ for $\lambda \in \Lambda \backslash\left\{\lambda^{\prime}\right\}$, and in this case $u_{J_{\lambda^{\prime}}} v_{J_{\lambda^{\prime}}} \in A\left(\prod_{\iota \in J_{\lambda^{\prime}}} G_{\iota}\right)$. This means that $u v$ is an $\operatorname{arc}$ in $H$ if it is an arc in $G$.

On the other hand, if we have an arc $u v$ in $H$, then $u_{J_{\lambda}}=v_{J_{\lambda}}$ for all $\lambda \in \Lambda$, except for one, say $\lambda^{\prime}$, for which $u_{J_{\lambda^{\prime}}} v_{J_{\lambda^{\prime}}} \in A\left(\prod_{\iota \in J_{\lambda^{\prime}}} G_{\iota}\right)$. But then $u_{\iota}=v_{\iota}$ for all $\iota \in J_{\lambda}$ for $\lambda \neq \lambda^{\prime}$, and in $J_{\lambda^{\prime}}$ there is a $\kappa$ such that $u_{\kappa} v_{\kappa} \in A\left(G_{\kappa}\right)$ and $u_{\iota} v_{\iota}$ for $\iota \in J_{\lambda^{\prime}} \backslash\{\kappa\}$, hence $u v \in A(G)$. Thus $u v$ is an arc in $G$ if it is an arc in $H$.

For the loops we observe that all $G$-coordinates of a vertex $v$ are unlooped if and only if all $H$-coordinates of $v$ are unlooped. Hence a vertex has no loop in $G$ if and only if it has no loop in $H$.

It is easily seen, and well known, that the Cartesian product of finitely many factors is connected if and only if all factors are connected, but the product of infinitely many nontrivial graphs is always disconnected. The reason is that such products contain vertices that differ in infinitely many coordinates, but every arc (which is not a loop) connects vertices that differ in exactly one coordinate, which means that the coordinates of any two vertices that are connected by a path can differ in only finitely many coordinates.

The connected components of the Cartesian product $\prod_{\iota \in I} G_{\iota}$ of infinitely many connected factors are called weak Cartesian products. We will use the notation $\prod_{\iota \in I}^{a} G_{\iota}$ to indicate the component that contains the vertex $a \in V\left(\prod_{\iota \in I} G_{\iota}\right)$.

For finite graphs, or for products of finitely many factors, the Cartesian product and the weak Cartesian product coincide.

\section{Unique prime factorization}

It is well known that simple, connected graphs have unique prime factorizations with respect to the weak Cartesian product; see [8, 6]. We complete this note by showing that this is also the case for directed graphs with loops. Our proof is direct, it does not use the result for undirected graphs. 
Theorem 5 Every connected, finite or infinite graph in $\overrightarrow{\Gamma_{0}}$ with at least one unlooped vertex has a unique prime factor decomposition with respect to the Cartesian or weak Cartesian product.

Proof Let $G$ be a connected graph in $\overrightarrow{\Gamma_{0}}$ and $a$ an unlooped vertex. Consider all possible representations of $G$ as products of two factors $A_{1, \kappa} \square A_{2, \kappa}, \kappa \in K$, and let $e$ be an arc incident with $a$. Observe that, for any $\kappa \in K$, exactly one of the layers $A_{1, \kappa}^{a}, A_{2, \kappa}^{a}$ contains $e$. We denote it by $A_{i_{e}, \kappa}^{a}$ and form

$$
G_{e}=\bigcap_{\kappa \in K} A_{i_{e}, \kappa}^{a} .
$$

Clearly $G_{e}$ is convex in $G$, because it is the intersection of convex subgraphs of $G$. Also, if $f$ is another arc incident with $a$, and if $G_{f} \neq G_{e}$, then there exist $\kappa \in K$ such that $A_{i_{f}, \kappa}^{a} \neq A_{i_{e}, \kappa}^{a}$. Then $G_{f} \cap G_{e} \subseteq A_{i_{f}, \kappa}^{a} \cap A_{i_{e}, \kappa}^{a}=a$. Hence, any two distinct $G_{f}, G_{e}$ have only the vertex $a$ in common.

We will show that $G_{e}^{a}$ is a factor of $G$, or, to be more precise, a unit layer with basepoint $a$ of a factorization of $G$, and that $G$ is the Cartesian or weak Cartesian product of all distinct $G_{e^{-}}$s. Note, if $G_{e}^{a}$ is a factor, then it must be prime, otherwise it would not be the intersection of all layers containing $e$, and if $P$ is a prime factor of $G$, and $e$ an arc incident with $a$ that projects to an arc of $P$, then $P^{a}=G_{e}$.

Hence, if $G_{\iota}, \iota \in I$, denotes the set of all distinct $G_{e^{-s}}$, and if we can show that

$$
G=\prod_{\iota \in I}^{a} G_{\iota},
$$

then this is the unique representation of $G$ as a Cartesian or weak Cartesian product.

We now define the coordinates of a vertex $v$ of $G$ as the vertices of shortest distance in the $G_{e^{-S}}$ from $v$. So the $G_{e}$-coordinate of $v \in G$ is the unique vertex of $G_{e}$ that is closest to $v$. Clearly all coordinates of $a$ are $a$.

Consider a vertex $v \neq a$. If $v$ is either in $A_{1, \kappa}^{a}$ or in $A_{2, \kappa}^{a}$ for all $\kappa \in K$, then $v$ is in $G_{e}$, where $e$ is an arc incident with $a$ on an arbitrary shortest path from $a$ to $v$. So it is identical to its coordinate in $G_{e}$. All other coordinates clearly are $a$. Otherwise there must be a $\kappa_{1} \in K$ such that $v \notin A_{i, \kappa_{1}}^{a}$, for $i \in\{1,2\}$. Let $v_{1}$ be the projection of $v$ into $A_{i_{1}, \kappa_{1}}^{a}$, where $i_{1}$ is chosen from $\{1,2\}$. Notice that $d_{G}\left(v_{1}, a\right)<d_{G}(v, a)$. If there is a $\kappa_{2}$ such that $v_{1} \notin A_{i, \kappa_{2}}^{a}$, for $i \in\{1,2\}$, then we form $v_{2}$ as the projection of $v_{1}$ into $A_{i_{2}, \kappa_{2}}^{a}, i_{2} \in\{1,2\}$. As before $d_{G}\left(v_{2}, a\right)<d_{G}\left(v_{1}, a\right)$, hence this process cannot continue indefinitely. It ends when, for some $k, v_{k}=a$, or when $v_{k}$ is either in $A_{1, \kappa}^{a}$ or in $A_{2, \kappa}^{a}$ for all $\kappa \in K$, and hence, by the previous argument, in a $G_{e}$, where $e$ is an $\operatorname{arc}$ incident with $a$ that is on a shortest path to $v_{k}$. Notice that $v \notin A_{i_{1}, \kappa_{1}}^{a}$ and that

$$
A_{i_{1}, \kappa_{1}}^{a} \supset A_{i_{2}, \kappa_{2}}^{a} \supset \cdots \supset A_{i_{k}, \kappa_{k}}^{a} \supseteq G_{e} .
$$

Hence, by Lemma 1 for any vertex $x \in G_{e}$ there is a shortest $v, x$-path that passes through $v_{1}$. Similarly, there is a shortest $v_{1}, x$-path through $v_{2}$, and finally through $v_{k}$. Hence, if $x$ is on a shortest path from $G_{e}$ to $v$, then it must be equal to $v_{k}$.

Thus, $v_{k}$ is the unique vertex of shortest distance from $G_{e}$ to $v$, and thus its $G_{e}$-coordinate. Note that, by the construction of $v_{k}$, every $v$ can have at most finitely many coordinates that are different from $a$.

We still have to show that different vertices have different coordinates. Let $u \neq v$, where $u \neq a$ and $e$ is an arc incident with $a$ on a shortest $a, u$-path. Suppose first that, for any $\kappa \in K$, the 
vertices $u$ and $v$ are either both in $A_{1, \kappa}^{a}$, or both in $A_{2, \kappa}^{a}$. Then $u$ and $v$ are both in $G_{e}$, and equal to their $G_{e}$ coordinates, which are different. If this is not the case, then there is a $\kappa \in K$, such that the projections of $u$ and $v$ into at least one of $A_{1, \kappa}^{a}$ or $A_{2, \kappa}^{a}$ are distinct and different from $\{u, v\}$, we call them $u_{1}, v_{1}$. We can continue this until both $u_{k}$ and $v_{k}$ are in the same $G_{e}$. But then the $G_{e}$-coordinate of $v$ is $a$ and different from the $G_{e}$-coordinate of $u$.

Having uniquely coordinatized the vertices of $G$, let us consider the projections of loops and arcs into the factors.

First the arcs. Let $u v$ be an $\operatorname{arc}$ in $G$. If $u v \in G_{e}$, then $u v$ is equal to its projection into $G_{e}$, and all the other projections, that is, coordinates, are $a$. Otherwise we proceed as above, when we showed that different vertices have different coordinates. We just have to observe that $u v$ is an arc if and only if $u_{1} v_{1}$ is an arc, and, by induction, that $u v$ is an arc if and only if the projections into $G_{e}$ are an arc. Furthermore, there is always only one projection we can choose from such that the $u_{i}, v_{i}$ remain different. So, if we alter any of the sequences of projections that sends $u$ into $G_{e}$, then $u, v$ end up in the same vertex in some other $G_{f}$, which means that they have the same $G_{f}$-coordinates.

Now the loops. Observe, if $v$ is unlooped, then this is also the case of $v_{1}, v_{2}, \ldots, v_{k}$, but if $v$ has a loop then one or both of the projections into $A_{1, \kappa_{1}}^{a}(v)$ or $A_{2, \kappa_{1}}^{a}(v)$ have a loop. We choose for $v_{1}$ a projection with a loop and continue like this. Then this process ends in a coordinate of $v$ that has a loop. In other words, $v$ is unlooped if and only if all projections are unlooped.

\section{References}

[1] T. Boiko, J. Cuno, W. Imrich, F. Lehner, Ch. E. van de Woestijne, The Cartesian product of graphs with loops, Ars Math. Contemp. 11 (2016), 1-9.

[2] Ch. Crespelle, E. Thierry, Computing the directed Cartesian-product decomposition of a directed graph from its undirected decomposition in linear time, Discrete Mathematics 338 (2015), 2393-2407.

[3] J. Feigenbaum, Directed cartesian-product graphs have unique factorizations that can be computed in polynomial time, Discrete Appl. Math. 15 (1986), 105-110.

[4] J. Feigenbaum, J. Hershberger, A. Schäffer, A polynomial time algorithm for finding the prime factors of cartesian-product graphs, Discrete Appl .Math. 12 (1985), 123-138.

[5] R. Hammack, W. Imrich, S. Klavžar, Handbook of Product Graphs (Second Edition), Taylor \& Francis Group (2011).

[6] W. Imrich, Über das schwache kartesische Produkt von Graphen, J. Combinatorial Theory Ser. B 11 (1971), 1-16.

[7] W. Imrich, I. Peterin, Recognizing cartesian products in linear time, Discrete Math. 307 (2007), $472-483$.

[8] D. J. Miller, The automorphism group of a product of graphs, Proc. Amer. Math. Soc. 25 (1970), 24-28. 
[9] G. Sabidussi, Graph multiplication, Math. Z. 72 (1959/1960), 446-457.

[10] V. G. Vizing, The cartesian product of graphs, (Russian) Vychisl. Sistemy 9 (1963), 30-43. 